\title{
Digital Control of Electric Power Flows in Unbalanced Distribution Networks as Part of The Automated Metering and Control System
}

\author{
T.T.Omorov ${ }^{1, *}$, B.K.Takyrbashev ${ }^{1 \cdot}$ K.E.Zakiriaev ${ }^{2}$, T.Zh.Koibagarov ${ }^{1}$
}

\begin{abstract}
${ }^{1}$ National Academy of Sciences of the Kyrgyz Republic, Institute of Mechanical Engineering and Automation, Bishkek
${ }^{2}$ Issyk-Kul State University named after K. Tynystanov, Karakol, Kyrgyz Republic
\end{abstract}

\begin{abstract}
This paper aims to address the problem of controlling the electricity flows in power distribution networks (PDN) operating under current and voltage unbalance. As is known, the unbalance factor is responsible for significant losses of active power and, therefore, is detrimental to the PDN efficiency and technical and economic performance. The purpose of control is to minimize technical power losses in the distribution network. This is to be achieved by building an information control system (ICS) for balancing a three-phase network as part of the automated metering and control system (AMCS). The latter is currently being widely adopted to automate information processes in PDNs. However, the AMCS does not include technologies for solving the problem in question. We propose an algorithm of the digital controller operation for the ICS. Its primary function is to maintain phase power at a given level in real-time. The algorithm concept is based on the idea of required redistribution of electricity flows between the phases of the distribution network by appropriately switching single-phase loads of consumers (users) to ensure a minimum spread of phase powers relative to their required level. To achieve the goal of control, we construct criterion functions that determine qualitative indices of the ICS operation and develop computational schemes for their minimization. Control actions to be generated by the digital controller and performed on the facility represent a digital code that contains data on the coordinates of threephase network loads to be switched to another phase.
\end{abstract}

Index Terms: distribution network, unbalanced mode, control of electricity flows, digital controller, control algorithm.

\footnotetext{
${ }^{*}$ Corresponding author.

E-mail: omorovtt@mail.ru
}

http://dx.doi.org/10.38028/esr.2021.01.0005

Received March 26, 2021. Revised April 03, 2021.

Accepted April 13, 2021. Available online May 26, 2021.

This is an open access article under a Creative Commons Attribution-NonCommercial 4.0 International License.

(C) 2021 ESI SB RAS and authors. All rights reserved.

\section{INTRODUCTION}

At present, power distribution networks (PDN) are widely adopting advanced digital technologies in the form of automated metering and control systems (AMCSs) to automate their processes and make them data-driven [1]. These systems are elements of the Smart Grid technology [2]. An analysis of the functional structure of existing (conventional) AMCSs shows that they mainly perform metering and fiscal metering of electricity and belong to the class of information and measurement systems. The latter lack tools designed to solve the problem of balancing three-phase distribution networks. At the same time, most of the $0.4 \mathrm{kV}$ power distribution networks operate under current and voltage unbalance [3-5], which causes nonoptimal operating conditions. Unbalance of PDN results from unbalance of loads and their uneven distribution over phases of a three-phase network. According to the published research data, in networks with distributed load, which have a deviation (unbalance) of phase currents from their average values, technical losses increase significantly [6-8]. The results of experimental studies of power supply systems indicate that technical losses in power lines and transformer substations (TS) supplying them amount to more than 6\% of the total electricity supplied to the inputs of distribution networks [9]. At the same time, the quality of electricity deteriorates, and the likelihood of failure of household appliances and industrial installations also increases [8]. Although many methods and devices were proposed [1013] for balancing distribution networks, their practical use proves difficult. This is due to the technical challenges of creating automated balancing systems on their basis for three-phase distribution networks with a large number of electricity consumers. For the technical implementation of these solutions, at points of load connection to the network phases, it is necessary to create separate local subsystems with individual units as their parts to perform functions of parameter measurement, control, and data processing. However, building such subsystems involves rather elaborate processing algorithms based on measurements of a group of information parameters. For example, the method outlined in [10] requires measurements of the active 
power of a zero wire of line, voltage and current signals of the zero wire at the points of load connection to the network, while the reactive power control system [13] uses positive sequence, negative sequence, and zero sequence currents of loads. In modern electricity meters [16], which are part of the AMCS, these measurement functions are not available. In this regard, the use of the above technologies as part of modern AMCSs seems to be problematic. In [14, 15], one of the possible approaches proposed to solve the given problem is based on a digital system for automatic control of the network balancing process as part of the data concentrator of the AMCS. As is known, a data concentrator in conventional automated systems collects data from a group of electricity meters installed at the side of network users and performs computing operations related to fiscal metering of electricity. According to this approach, the data concentrator is assigned with an additional function of digital control, which provides the required redistribution of electricity flows between the network phases by switching single-phase loads from a more loaded phase to a less loaded one. At the same time, electricity meters must have dedicated actuating elements, i.e., phase current switches (PCS), as their part. The creation of such PCSs is a feasible task since modern single-phase and three-phase meters $[16,17]$ have relays with powerful contacts up to $100 \mathrm{~A}$, which can be used to switch power consumers from one phase to another [15].

The present study aims to develop this approach for solving problems of control of electricity flows in unbalanced distribution networks.

\section{PROBLEM STATEMENT}

Let us consider a three-phase four-wire distribution network to which a power supply transformer substation (TS) and a group of $n$ electricity meters $\left\{E m_{v k}\right\}$ installed at singlephase consumers are connected. Its simplified diagram is shown in Fig. 1, where $k, v$ are index variables denoting, respectively, the numbers of phases $A, B, C(k=\overline{1,3})$ and loads (electrical consumers) of network users $(v=1, n) ; \tilde{I}_{k}$, $\tilde{U}_{k}$ are sinusoidal instantaneous current and voltage at the input of the $k$-th phase; $Z_{v k}$ is the load with the coordinate $(v, k) ; \tilde{I}_{v k}, \tilde{U}_{v k}$ are instantaneous current and voltage across the load $Z_{v k} ; \tilde{J}$ is instantaneous current in the zero wire; $\dot{P}_{k}$ is complex power of the $k$-th phase; $\dot{P}_{v k}$ is complex power consumed by the consumer load with the coordinate $(v, k)$; $P C S_{v k}$ is a switch of phase currents of the meter $E m_{v k}$.

Next, we make the following assumptions:

1. the three-phase network operates under current and voltage unbalance;

2. the phase current switches $\left(P C S_{v k}\right)$ are a part of the electricity meters $\left(E m_{v k}\right)$;

3. the concentrator at discrete moments of time $t=t_{\xi}$ $(\xi=1,2, \ldots)$ periodically polls the head-end three-phase electricity meter $\left(E m^{T S}\right)$ and groups of consumers' meters connected to phases $A, B, C$, and writes to the database AMCS values of the following quantities:

- active $p_{v k}$ and reactive $q_{v k}$ power consumed by loads of network users with coordinates $(v, k)$;

- active $p_{k}$ and reactive $q_{k}$ powers consumed by the corresponding phases at the input of the three-phase network.

TS

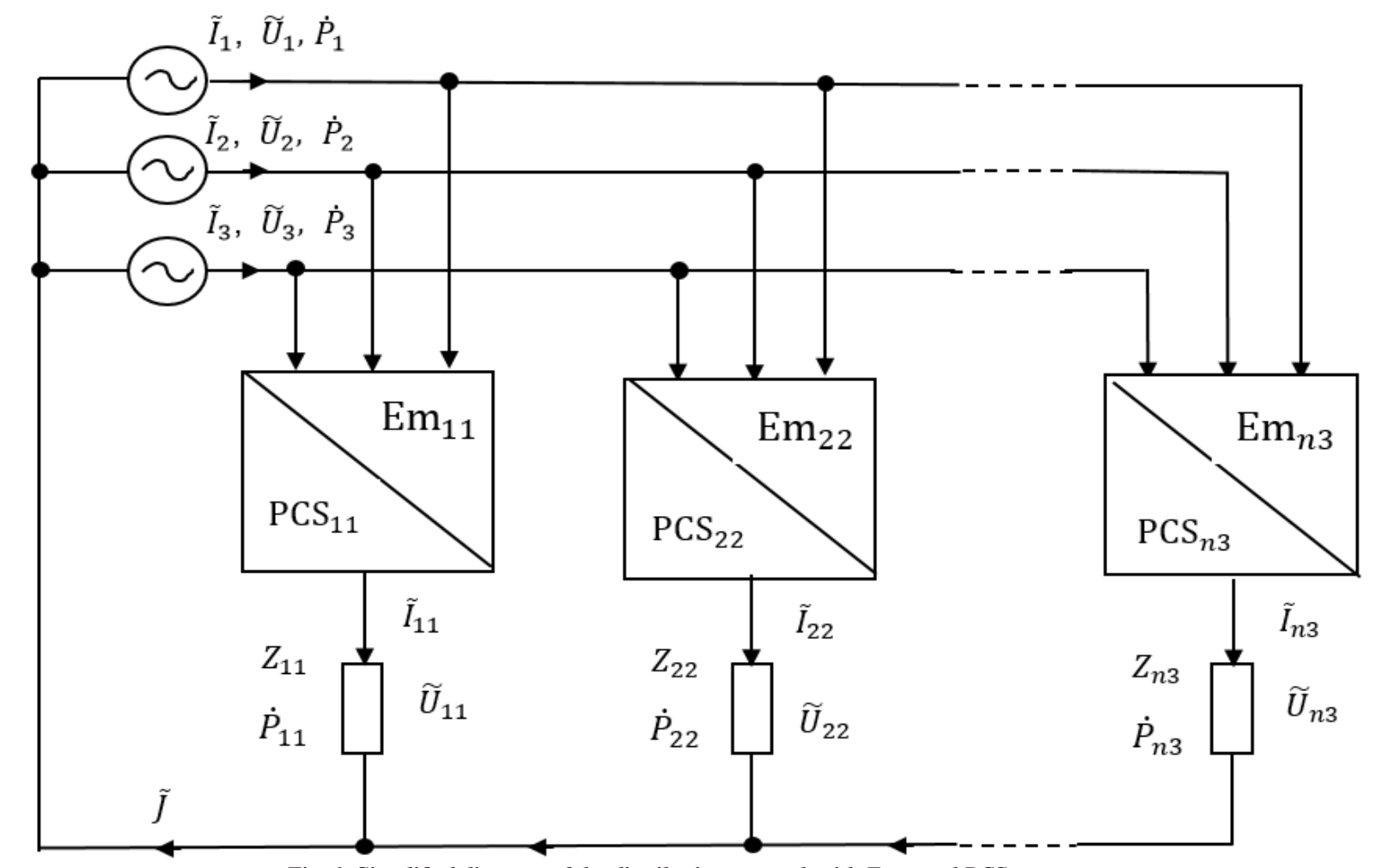

Fig. 1. Simplified diagram of the distribution network with Em $\mathrm{Fin}_{v \mathrm{PCS}}$ 
Technical losses of electricity in the transformer substation and the network are largely determined by the value of current $J$ flowing at the head-end of the zero wire. Moreover, the lower the value of this current, the higher the level of the network balance and the lower the power loss there. Therefore, the quality and efficiency of the distribution network can be assessed by the following objective function [15]:

$$
E=J .
$$

Minimizing the efficiency index of system $E$ is equivalent to optimizing the operating condition of an unbalanced distribution network, which provides a minimum unbalance in the values of the total power consumed by each phase of the network. To this end, we propose creating an appropriate information control system (ICS) as part of the modern (conventional) AMCS. The development of the ICS is to be based on the principles of building automatic systems [18] and digital technologies, the functional structure of which is shown in Fig. 2. In this case, the group of loads $\left\{Z_{v k}\right\}$ of network consumers to which the electricity meters $\left\{E m_{v k}\right\}$ are connected, together with the

ICS actuating elements, is a control object. The key functional block of the system is a digital controller (DC) based on a microprocessor controller $[19,20]$. The DC block generates control actions $u^{*}$ to be performed on the facility based on a dedicated algorithm (control rule). The DC operation program is built by the automatic system initial state identifier (ISI) as setting action $\rho^{*}$. In this case, the control signal $u^{*}$ is a digital code of the command, which is formed as vector $F=\left[F_{1}, F_{2}, \beta\right]$, where $F_{1}, F_{2}$ are numbers (names) of a pair of phases in which it is necessary to switch network consumers with a more loaded phase $\left(F_{1}\right)$ to a less loaded one $\left(F_{2}\right) ; \beta$ is a vector composed of addresses (numbers) of users of phase $F_{1}$ to be switched. This control signal $u^{*}$ is transmitted via the communication channel (CC) to the system actuating elements, which are used as phase current switches (PCS) designed to perform the required switching of the network users' loads from one phase to another [15]. Conventional AMCSs employ various data transmission technologies (PLC, GSM, and others) as the CC.

The task is to synthesize an algorithm for the operation of a digital controller that optimizes the functioning of an unbalanced distribution network.

\section{THE CONTROL PROBLEM}

The above control problem-solving process includes the following main steps:

1. Define the input data of the control problem.

2. Conduct a case study of the facility.

3 . Construct criterion functions.

4. Build an algorithm for minimizing criterion functions.

5. Generate control actions to be performed on the facility.

\section{A. Define the input data of the problem}

Based on the data obtained from the main three-phase meter and consumer meters, the complex powers $\dot{P}_{k}$ and $\dot{P}_{v k}$, respectively, at the network input and consumer loads, are represented as [21]:

$$
\begin{gathered}
\dot{P}_{k}=p_{k}+j q_{k}, \quad k=\overline{1,3}, \\
\dot{P}_{v k}=p_{v k}+j q_{v k}, v=\overline{1, n}, k=\overline{1,3}, j=\sqrt{-1},
\end{gathered}
$$

Where $\mathrm{j}$ is an imaginary number. In this case, absolute values of these complex quantities are determined by the expressions:

$$
\begin{gathered}
P_{k}=\sqrt{p_{k}^{2}+q_{k}^{2}}, \quad k=\overline{1,3}, \\
P_{v k}=\sqrt{p_{v k}^{2}+q_{v k}^{2}}, \quad v=\overline{1, n}, \quad k=\overline{1,3} .
\end{gathered}
$$

It is worth noting that to solve the above-stated control problem, it is sufficient to poll only a small group of single-phase consumer electricity meters, except for meters installed at two-phase consumers. Further, we denote by $n_{1}, n_{2}$, and $n_{3}$ the total number of simplified consumer meters connected to phases $A, B$, and $C$, respectively. For brevity of notation and convenience of further transformations, the data on absolute values of complex powers of network consumers, determined as per

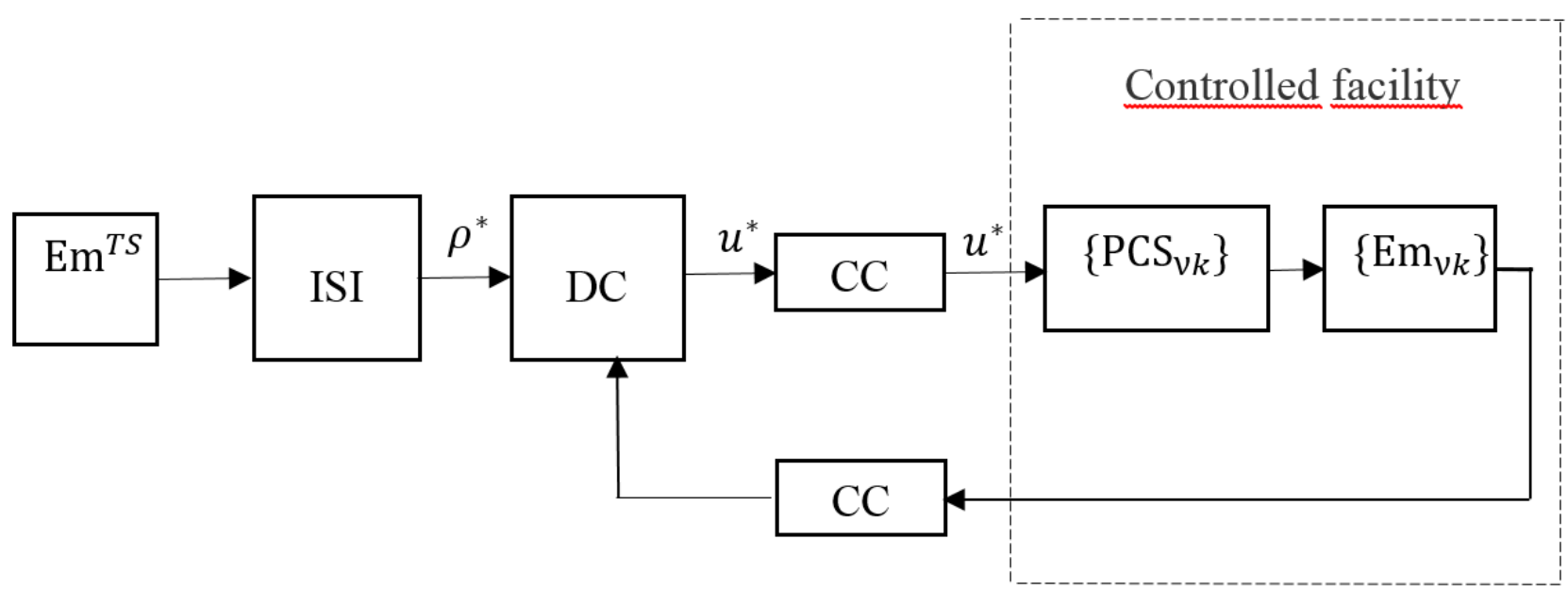

Fig. 2. ICS functional structure. 
formulas (3), are provisionally presented in the form of the following vectors:

$$
\begin{aligned}
P^{A} & =\left[P_{1}^{A}, P_{2}^{A}, \ldots, P_{n_{1}}^{A}\right], \\
P^{B} & =\left[P_{1}^{B}, P_{2}^{B}, \ldots, P_{n_{2}}^{B}\right], \\
P^{C} & =\left[P_{1}^{C}, P_{2}^{C}, \ldots, P_{n_{3}}^{C}\right],
\end{aligned}
$$

and the coordinates of their elements are set by vectors:

$$
\begin{aligned}
& \beta^{A}=\left[\beta_{1}^{A}, \beta_{2}^{A}, \ldots, \beta_{n_{1}}^{A}\right], \\
& \beta^{B}=\left[\beta_{1}^{B}, \beta_{2}^{B}, \ldots, \beta_{n_{2}}^{B}\right], \\
& \beta^{C}=\left[\beta_{1}^{C}, \beta_{2}^{C}, \ldots, \beta_{n_{3}}^{C}\right],
\end{aligned}
$$

where superscripts $A, B$, and $C$ indicate that the introduced vectors and their elements refer to the corresponding phases of the three-phase network. When forming vectors $P^{A}, P^{B}$, $P^{C}$, their components can be positioned arbitrarily. In this case, the set of components of indicated vectors make up the sets $\Lambda_{A}, \Lambda_{B}, \Lambda_{C}$, respectively:

$$
\Lambda_{A}=\left\{P_{v}^{A}\right\}_{1 \times n_{1}}, \Lambda_{B}=\left\{P_{v}^{B}\right\}_{1 \times n_{2}}, \Lambda_{C}=\left\{P_{v}^{C}\right\}_{1 \times n_{3}} .
$$

\section{B. Conduct the case study of the facility}

Such a study is performed to determine the structure of phase switching, i.e., to identify names of phases $\left(F_{1}, F_{2}\right)$, in which corresponding loads of network consumers are to be switched. To this end, the average value $P_{0}$ of the phase power absolute values is provisionally calculated based on the formulas (2):

$$
P_{0}=\left(P_{1}+P_{2}+P_{3}\right) / 3 \text {. }
$$

Similarly, one can determine the average value of effective phase currents $I_{1}, I_{2}$, and $I_{3}$ at the network input:

$$
I_{0}=\left(I_{1}+I_{2}+I_{3}\right) / 3 \text {. }
$$

To assess the level of unbalance (deviations) of phase powers and currents from their average values, we introduce the following differences (residuals) into consideration:

$$
\begin{aligned}
& e_{k}=P_{k}-P_{0}, \quad k=\overline{1,3}, \\
& \varepsilon_{k}=I_{k}-I_{0}, \quad k=\overline{1,3} .
\end{aligned}
$$

Note that the values $e_{1}, e_{2}$, and $e_{3}$, as determined by formulas (5), are essentially control errors (residuals) that should tend to zero during control. In this case, current absolute values of phase powers at the input of the distribution network are determined by the formulas

$$
P_{k}=P_{0}+e_{k}, k=\overline{1,3} \text {. }
$$

Hence one can see that at $e_{k} \rightarrow 0$ phase powers are $P_{k} \rightarrow P_{0}, \quad k=\overline{1,3}$. Thus, the problem of optimizing the operating condition of the distribution network in each interval $\left[t_{\xi}, t_{\xi+1}\right]$ is reduced to minimizing control errors $e_{1}$, $e_{2}$, and $e_{3}$. In this case, control actions $u^{*}$, when performed on the facility, also lead to desired changes in effective phase currents $I_{1}, I_{2}$, and $I_{3}$ at the network inputs. Indeed, by definition, complex powers $P_{k}$ at inputs of corresponding phases are determined by the formulas [21]:

$$
\dot{P}_{k}=\dot{U}_{k} \dot{I}_{k}^{*}=I_{k}^{2} z_{k}, \quad k=\overline{1,3},
$$

where $\dot{U}_{k}, \dot{I}_{k}$ are complex voltage and current at the input of the $k$-th phase; $\dot{I}_{k}^{*}$ is a complex conjugate value corresponding to current $\dot{I}_{k} ; z_{k}$ is complex resistance of the $k$-th phase. Therefore, the following expressions can be written for absolute values:

$$
P_{k}=I_{k}^{2} Z_{k}, k=\overline{1,3},
$$

where $Z_{k}$ is the absolute value of complex impedance $z_{k}$. Therefore, for effective phase currents $I_{k}$, the following formulas are valid:

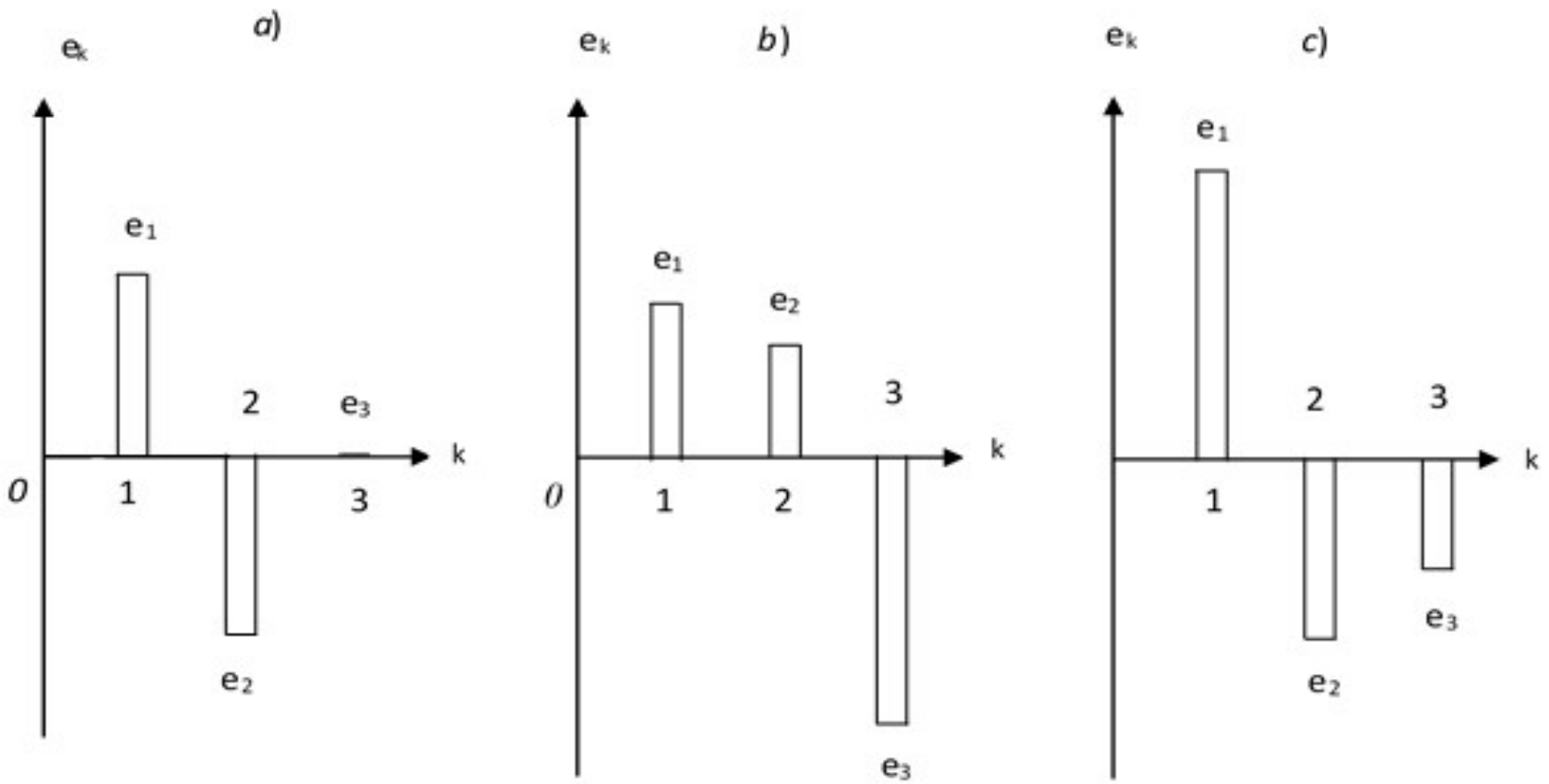

Fig. 3. Illustration of possible variants of control errors $e_{k}$. 


$$
I_{k}=\sqrt{P_{k} / Z_{k}}, \quad k=\overline{1,3} .
$$

It is worth noting here that the connection of a new load to the selected phase leads to an increase in the magnitude of the absolute value of power $P_{k}$ and a decrease in its impedance $Z_{k}$. Therefore, in this case, the ratio $P_{k} / Z_{k}$ increases, and when the load is disconnected, the value of $P_{k} / Z_{k}$ decreases. Thus, in the process of control, an increase or decrease in the absolute values of power $P_{1}, P_{2}$, and $P_{3}$ leads to the same changes in the dynamics of operating phase currents $I_{1}, I_{2}$, and $I_{3}$.

In this case, corresponding absolute values of residuals for phase currents $\left|\varepsilon_{1}\right|,\left|\varepsilon_{2}\right|$, and $\left|\varepsilon_{3}\right|$ decrease so that the minimum of their unbalance is ensured. As a result, along with the equalization (balancing) of phases in terms of power, control of electricity flows in the distribution network also makes phase currents get balanced, which leads to the minimization of effective current $J$ flowing in the head-end of the zero wire of the three-phase network.

Based on the results of calculating the residuals $e_{k}$, $k=\overline{1,3}$, the following cases can be distinguished:

Case 1. One of the components of the control error vector has a positive value $e=\left[e_{1}, e_{2}, e_{3}\right]$, one of them is negative, and one of them is equal to zero. For example, $e_{1}>0, e_{2}<0, e_{3}=0$, which is shown in Fig. 3a. In this case, phase $A$ is overloaded, therefore, it is necessary to switch its consumers to phase $B$, which we denote further by $A \rightarrow B\left(F_{1}=\mathrm{A}, F_{2}=\mathrm{B}\right)$. In this case, phase $C$ should retain the previous state since $e_{3}=0$.

Case 2. Two components of the residual vector e are positive, and the third one has a negative value. For example, $e_{1}>0, e_{2}>0, e_{3}<0$ (Fig. 3b). In this case, phases $A$ and $B$ are overloaded. It is, therefore, necessary to switch $A \rightarrow C$ and $B \rightarrow C$.

Case 3. One of the elements of vector e is positive, and the rest are negative. For example, $e_{1}>0, e_{2}<0, e_{3}<0$ (Fig. 3c). In this case, it is necessary to switch $A \rightarrow B$ and $A \rightarrow C$.

Thus, to identify the required switchings of the distribution network phases, it is necessary to perform a case study of the signs of values of $e_{k}, k=\overline{1,3}$. In this case, it is necessary to switch some consumers from phases with positive values of errors $e_{k}$ to those with negative signs of control errors.

As a result, the case study of the facility provides for the following operations:

1. Poll the head-end three-phase electricity meter $\left(E m^{T S}\right)$ and a given set of meters installed at network consumers connected to phases $A, B, C$, and define input data of the control problem.

2. Form vector $P=\left[P_{1}, P_{2}, P_{3}\right]$, consisting of the absolute values of complex powers consumed by phases $A, B$, and $C$, according to formulas (2).

3. Calculate average value $P_{0}$ of the phase power absolute values, as determined by formula (4).
4. Determine values and signs of the components of the vector of control errors $e=\left[e_{1}, e_{2}, e_{3}\right]$ as per formulas (5).

5. Identify names of phases $\left(F_{1}, F_{2}\right)$, in which load switching operations are necessary, based on the analysis of signs of control error vector components $e=\left[e_{1}, e_{2}, e_{3}\right]$.

\section{Construct the criterion function}

It is easy to show that the initial state of the facility as it manifests itself by the components of the control error vector $e=\left[e_{1}, e_{2}, e_{3}\right]$ satisfies the following condition:

$$
e_{1}+e_{2}+e_{3}=0 \text {. }
$$

Next, without loss of generality, consider Case 1 at the facility. In the case under consideration, the synthesized controller must output the initial mismatch $\rho^{*}=\rho_{1}^{*}$ through the control error, which is formed by the ISI block. Its value can be determined from condition (6). Since following the considered Case the residual $e_{3}=0$, condition (6) will have the form

$$
e_{1}=\left|e_{2}\right| \text {. }
$$

Therefore, we arrive at $\rho_{1}^{*}=|e 2|$. Next, we form subset $\lambda_{1}$ consisting of a set of elements of the set $\Lambda_{A}$, i.e., $\lambda_{1} \in \Lambda_{A}$. In particular, $\lambda_{1}$ can consist of only one element. Let $R_{1}$ denote subset of indices (numbers) of vector components $\beta^{A}: R_{1}=\left\{1,2, \ldots, n_{1}\right\}$.

Now let us introduce the following function:

$$
F_{1}\left(\lambda_{1}\right)=\left|S_{1}\left(\lambda_{1}\right)-\rho_{1}^{*}\right|,
$$

where $S_{1}\left(\lambda_{1}\right)$ is a discrete function determined by the sum of elements of subset $\lambda_{1}$ :

$$
S_{1}\left(\lambda_{1}\right)=\sum_{l \in R_{1}} P_{l}^{A}
$$

The function $F_{1}\left(\lambda_{1}\right)$ defined by formula (7) can be used to indirectly estimate the value of the objective function $E$ defined by expression (1). The analysis shows that when $F_{1}\left(\lambda_{1}\right) \rightarrow 0$, the control errors $e_{1}$ and $e_{2}$ also tend to zero when switching consumers with more loads connected to the first phase (phase $A$ ).

\section{$D$. The algorithm for minimizing criterion functions}

Now it can be seen that in the case under consideration, coordinates of loads of phase $A$ users can be identified by solving the following extremal problem:

$$
\min _{\lambda_{1} \in \Lambda_{A}} F_{1}\left(\lambda_{1}\right)=F_{1}\left(\lambda_{1}^{*}\right)
$$

where $\lambda_{1}^{*}$ is the optimal set of absolute values of complex power consumed by phase $A$ loads $(k=1)$ to be switched

to phase $B$. The set $\lambda_{1}^{*}$ found this way contains complete information about the coordinates of consumer loads to be switched.

The minimization problem (8) is discrete and can be solved by enumerating options, which leads to timeconsuming computational operations and inadequate efficiency of the procedure for generating control actions to be performed on the facility. The analysis shows that a 
simpler and more efficient scheme for solving the extremal problem (8) can be constructed by transforming the input data of the problem in a certain way and constructing criterion functions so that in the process of solving it, a purposeful movement towards the optimum is provided by analogy with the gradient method [22]. For this purpose, we propose a computational procedure. First, we preliminarily transform input data of the problem, represented by power vectors $P^{A}, P^{B}$, and $P^{C}$. Such a transformation involves sorting the latter to arrange their components in ascending order. In this case, new vectors $\hat{P}^{A}, \hat{P}^{B}$, and $\hat{P}^{C}$ with the same dimensions as the original ones are formed:

$$
\begin{aligned}
\hat{P}^{A} & =\left[\hat{P}_{1}^{A}, \hat{P}_{2}^{A}, \ldots, \hat{P}_{n_{1}}^{A}\right], \\
\hat{P}^{B} & =\left[\hat{P}_{1}^{B}, \hat{P}_{2}^{B}, \ldots, \hat{P}_{n_{2}}^{B}\right], \\
\hat{P}^{C} & =\left[\hat{P}_{1}^{C}, \hat{P}_{2}^{C}, \ldots, \hat{P}_{n_{3}}^{C}\right],
\end{aligned}
$$

where

$$
\begin{gathered}
\hat{P}_{v-1}^{A} \leq \hat{P}_{v}^{A}, \hat{P}_{\xi-1}^{B} \leq \hat{P}_{\xi}^{B}, \hat{P}_{\eta-1}^{C} \leq \hat{P}_{\eta}^{C}, \\
v=\overline{2, n_{1}}, \quad \xi=\overline{2, n_{2}}, \eta=\overline{2, n_{3}} .
\end{gathered}
$$

In this case, new coordinates of the components of the indicated vectors are formed:

$$
\begin{aligned}
& \hat{\boldsymbol{\beta}}^{A}=\left[\hat{\boldsymbol{\beta}}_{1}^{A}, \hat{\boldsymbol{\beta}}_{1}^{A}, \ldots, \hat{\boldsymbol{\beta}}_{n_{1}}^{A}\right], \\
& \hat{\boldsymbol{\beta}}^{B}=\left[\hat{\boldsymbol{\beta}}_{1}^{B}, \hat{\boldsymbol{\beta}}_{2}^{B}, \ldots, \hat{\boldsymbol{\beta}}_{n_{2}}^{B}\right], \\
& \hat{\boldsymbol{\beta}}^{C}=\left[\hat{\boldsymbol{\beta}}_{1}^{C}, \hat{\boldsymbol{\beta}}_{2}^{C}, \ldots, \hat{\boldsymbol{\beta}}_{n_{2}}^{C}\right] .
\end{aligned}
$$

A simple analysis shows that to define functions $F_{1}\left(\lambda_{1}\right)$, depending on a situation at the facility, it is more expedient to use components of new vectors $\hat{P}^{A}, \hat{P}^{B}$, and $\hat{P}^{C}$. Now let us consider Case 1 of the facility, residuals are $e_{1}>0$, $e_{2}<0$, and error is $e_{3}=0$. In this case, the criterion function $F_{1}\left(\lambda_{1}\right)$ must be specified in the form

$$
F_{1}\left(\lambda_{1}\right)=F_{1}(r)=\sum_{l=1}^{r} \hat{P}_{l}^{A},
$$

where $\mathrm{r}$ is some integer $(r \geq 1)$.

As a result, problem (8) will be written as

$$
\min _{r \in R_{1}} F_{1}(r)=F_{1}\left(r^{*}\right) \text {. }
$$

Thus, the original problem is reduced to determining integer $r=r^{*}$ at which the optimum criterion function $F_{1}\left(\lambda_{1}\right)$ is achieved.

By solving (10), we identify vector $\beta^{*}$ that consists of addresses of loads of phase $A$ users to be switched to phase $B$. Vector $\beta^{*}$ found in this way is a truncated part of original vector $\hat{\beta}^{A}=\left[\hat{\beta}_{1}, \hat{\beta}_{2}, \ldots, \hat{\beta}_{n_{1}}\right]$, that is, $\beta^{*}=\left[\hat{\beta}_{1}, \hat{\beta}_{2}, \ldots, \hat{\beta}_{r^{*}}\right]$. In this case, the desired control vector $u^{*}$ has the following form:

$$
u^{*}=\left[A, B, \beta^{*}\right]=\left[1,2, \hat{\beta}_{1}, \hat{\beta}_{2}, \ldots, \hat{\beta}_{r^{*}}\right] .
$$

The above algorithm for solving the control problem can also be used for cases where the facility operation corresponds to Case 2 and Case 3. Consider the features of constructing algorithms of the functioning of a digital controller for these cases.
Suppose that Case 2 is observed in the controlled facility. Without loss of generality, we assume that the control errors are $e_{1}>0, e_{2}>0$, and $e_{3}<0$. In this case, condition (6) will be written in the form

$$
e_{1}+e_{2}=\left|e_{3}\right| \text {. }
$$

In this case, it is necessary to carry out two switchings $A \rightarrow C$ and $B \rightarrow C$. Therefore, based on the data received from the consumer electricity meters, we will form two vectors of the input data:

$$
\begin{aligned}
P^{A} & =\left[P_{1}^{A}, P_{2}^{A}, \ldots, P_{m_{1}}^{A}\right], \\
P^{B} & =\left[P_{1}^{B}, P_{2}^{B}, \ldots, P_{m_{2}}^{B}\right],
\end{aligned}
$$

and two vectors of their coordinates:

$$
\begin{gathered}
\beta^{A}=\left[\beta_{1}^{A}, \beta_{2}^{A}, \ldots, \beta_{m_{1}}^{A}\right], \\
\beta^{B}=\left[\beta_{1}^{B}, \beta_{2}^{B}, \ldots, \beta_{m_{2}}^{B}\right],
\end{gathered}
$$

where $m_{1}, m_{2}$ are dimensions of corresponding vectors.

Next, components of vectors $P^{4}$ and $P^{B}$ are sorted similarly to the previous case, i.e., new vectors $\hat{P}^{A}$ and $\hat{P}^{A}$ are formed with their components arranged in ascending order:

$$
\begin{aligned}
& \hat{P}^{A}=\left[\hat{P}_{1}^{A}, \hat{P}_{2}^{A}, \ldots, \hat{P}_{m_{1}}^{A}\right], \\
& \hat{P}^{A}=\left[\hat{P}_{1}^{A}, \hat{P}_{2}^{A}, \ldots, \hat{P}_{m_{1}}^{A}\right],
\end{aligned}
$$

Now, as previously, subsets $R_{2}$ and $R_{3}$ are introduced for the indices of vectors $\hat{\beta}^{A}$ and $\hat{\beta}^{B}$, respectively, i.e., $R_{2}=\left\{1,2, \ldots, m_{1}\right\}$ and $R_{3}=\left\{1,2, \ldots, m_{2}\right\}$.

Following condition (6), the levels of mismatches $\rho_{2}^{*}$ and $\rho_{3}^{*}$ for the absolute values of corresponding complex powers of switched phases $A$ and $B$ are set as follows:

$$
\rho_{2}^{*}=e_{1}, \rho_{3}^{*}=e_{2} \text {. }
$$

Now, to find the required coordinates of network consumer loads, it is necessary to carry out two types of switchings, $A \rightarrow C$ and $B \rightarrow C$. In this case, the following two criterion functions are constructed:

$$
\begin{gathered}
F_{2}\left(\lambda_{2}\right)=\left|S_{2}\left(\lambda_{2}\right)-\rho_{2}^{*}\right|, \\
F_{3}\left(\lambda_{3}\right)=\left|S_{3}\left(\lambda_{3}\right)-\rho_{3}^{*}\right|,
\end{gathered}
$$

Where $\lambda_{2}, \lambda_{3}$ are subsets composed of components of vectors $\hat{P}^{A}$ and $\hat{P}^{B}$, respectively, i.e., $\lambda_{2} \in \Lambda_{A}, \lambda_{3} \in \Lambda_{B}$. In this case, functions $S_{2}\left(\lambda_{2}\right)$ and $S_{3}\left(\lambda_{3}\right)$ are determined using the components of vectors $\hat{P}^{A}$ and $\hat{P}^{B}$ similarly to the previous case (Case 1) according to (9):

$$
\begin{aligned}
& S_{2}\left(\lambda_{2}\right)=S_{2}(\eta)=\sum_{\ell=1}^{\eta} \hat{P}_{\ell}^{A}, \\
& S_{3}\left(\lambda_{3}\right)=S_{3}(\theta)=\sum_{\gamma=1}^{\theta} \hat{P}_{\gamma}^{B} .
\end{aligned}
$$

Further, to find the desired coordinates of the loads connected to phases $A$ and $B$, two extremal problems are solved:

$$
\min _{\lambda \in R_{2}} F_{2}(\eta)=F_{2}\left(\eta^{*}\right)
$$




$$
\min _{\theta \in R_{3}} F_{3}(\theta)=F_{3}\left(\theta^{*}\right),
$$

where $\eta^{*}$ and $\theta^{*}$ are the optimal quantities of absolute values of complex powers consumed by loads of consumers of phases $A$ and $B$, respectively.

Extremal problems (11) and (12) are solved using the above algorithm for minimizing the criterion function $\mathrm{F} 1(\lambda 1)$. Since in the considered case it is necessary to carry out two types of switchings $\mathrm{A} \rightarrow \mathrm{C}$ and $\mathrm{B} \rightarrow \mathrm{C}$, then solving the last extremal problems, we obtain vector $\mathrm{u}^{*}=[\mathrm{u} 1, \mathrm{u} 2]$, where $u_{1}=\left[A, C, \beta_{1}^{*}\right], u_{2}=\left[B, C, \beta_{2}^{*}\right]$, $\beta_{1}^{*}=\left[\hat{\beta}_{1}^{A}, \hat{\beta}_{2}^{A}, \ldots, \hat{\beta}_{\eta^{*}}^{A}\right], \beta_{2}^{*}=\left[\hat{\beta}_{1}^{B}, \hat{\beta}_{2}^{B}, \ldots, \hat{\beta}_{\theta^{*}}^{B}\right]$.

When Case 3 is observed at the facility and, for example, errors are $e_{1}>0, e_{2}<0, e_{3}<0$, condition (6) takes the form

$$
e_{1}=\left|e_{2}\right|+\left|e_{3}\right| .
$$

In this case, it is necessary to carry out switchings $A \rightarrow B$ and $A \rightarrow C$. Therefore, two vectors are formed from the set $\Lambda_{A}$ :

$$
\begin{aligned}
& P^{A}=\left[P_{1}^{A}, P_{1}^{A}, \ldots, P_{m_{1}}^{A}\right], \\
& \tilde{P}^{A}=\left[\tilde{P}_{1}^{A}, \tilde{P}_{1}^{A}, \ldots, \tilde{P}_{m_{2}}^{A}\right],
\end{aligned}
$$

as well as vectors $\beta^{A}$ and $\tilde{\beta}^{A}$, respectively, for their coordinates. Moreover, vectors $P^{A}$ and $\tilde{P}^{A}$ must have different components. Respectively, the components of these vectors form sets that do not intersect in this case. Next, we form the subsets $\lambda_{4} \in \Lambda_{A}$ and $\lambda_{5} \in \tilde{\Lambda}_{A}$. These input data are used in the above computational scheme to determine the desired control vector $u^{*}$. Firstly, by sorting components of vectors $P^{4}$ and $P^{4}$, new vectors $\hat{P}^{A}$ and $\tilde{P}^{A}$ are formed with their components arranged in ascending order. Then, criterion functions $F_{4}\left(\lambda_{4}\right)$ and $F_{5}\left(\lambda_{5}\right)$ are determined:

$$
\begin{aligned}
& F_{4}\left(\lambda_{4}\right)=\left|S_{4}\left(\lambda_{4}\right)-\rho_{4}^{*}\right|, \\
& F_{5}\left(\lambda_{5}\right)=\left|S_{5}\left(\lambda_{5}\right)-\rho_{5}^{*}\right|,
\end{aligned}
$$

where $\rho_{4}^{*}=\left|e_{2}\right|$ and $\rho_{5}^{*}=\left|e_{3}\right|$, and the functions $S_{4}\left(\lambda_{4}\right)$ and $S_{5}\left(\lambda_{5}\right)$ are defined similarly to the previous case. As in previous cases, the desired control vector $u^{*}$ is determined based on minimizing criterion functions $F_{4}\left(\lambda_{4}\right)$ and $F_{5}\left(\lambda_{5}\right)$. In this case, the algorithm for solving the extremal problem (10) is used.

To improve the ICS reliability and extend the service life of the phase current switch (PCS), it is advisable to limit the number of switchings of consumer electricity meters. To this end, a current sensor installed at the transformer substation can be used, the output of which is fed to a digital controller (DC). Based on the value of current $J$ of zero wire, DC checks the balance (quasioptimality) condition of the system in each control interval. This condition, in particular, can be specified by the following inequality:

$$
J \leq \Delta,
$$

where $\Delta$ is the maximum permissible value of current $J$, at which permissible level of the distribution network balance is ensured. To limit the number of switchings of consumer meters, control actions are generated and implemented only when condition (13) is not met.

The ICS constructed in this way with a digital controller can be considered as a system of a variable structure [23] since this will ensure the adaptation of the three-phase distribution network to the conditions that arise during uncontrolled random changes in its loads by automatic self-adjustment of its structure in real-time.

Based on the results obtained above, one can formulate a generalized algorithm of the ICS operation, which includes the following basic operations:

1. Poll the main three-phase electricity meter $\left(\mathrm{Em}^{T S}\right)$ and a given set of single-phase consumer meters connected to phases $A, B$, and $C$, except for meters installed at two-phase consumers.

2. Measure effective current $J$ of zero wire and verify condition (13). If it is not met, go to step 3. Otherwise, the DC block does not generate control commands for the facility.

3. Formulate the input data of the control problem determined by vectors $P, P^{A}, P^{B}, P^{C}, \beta^{A}, \beta^{B}, \beta^{C}$.

4. Estimate components of control error vector $e=\left[e_{1}, e_{2}, e_{3}\right]$ by formulas (5).

5. Conduct a case study of the facility based on the signs of components of the control error vector $e=\left[e_{1}, e_{2}, e_{3}\right]$ and identify an interphase switching structure determined by vector $F=\left[F_{1}, F_{2}\right]$.

6. Determine the initial mismatch $\rho^{*}$ by the ICS unit and construct corresponding criterion functions $\{\mathrm{Fi}(\lambda)\}$ depending on the identified situation at the facility. For example, for Case 1 at the facility $\rho^{*}=\rho_{1}^{*}=\left|e_{2}\right|$ , and $F_{1}\left(\lambda_{1}\right)$ is constructed as a criterion function.

7. Minimize selected criterion functions $\left\{F_{i}(\lambda)\right\}$. In this case, the DC block solves the extremal problems (10) or (11), (12), depending on the situation at the facility.

8. Generate digital control commands $u^{*}$ and transfer them to corresponding phase current switches to implement the required switching of the selected loads (meters) of the distribution network users.

\section{CONCLUSION}

Most three-phase distribution networks operate under unbalanced currents and voltages due to the unbalance of loads and their uneven distribution over phases. The unbalance factor causes an increase in active power losses in networks and transformer substations, which significantly diminishes the technical and economic performance of distribution companies.

At the same time, modern AMCS systems do not solve the problem of electricity flow control in unbalanced distribution networks as they focus on optimization of their operating conditions. We have proposed a novel approach to the construction of an information control system (ICS) aimed at solving this problem as part of a conventional AMCS system. The main idea, in this case, is the distribution 
of electricity flows between the phases of the network by switching its consumers from a more loaded phase to a less loaded one. To assess the quality of control processes, we have introduced criterion functions that characterize technical losses of electricity in the transformer substation and distribution network. We have proposed computational schemes for constructing them in terms of the situation at the facility. We have developed algorithms of operation of ICS of digital controller and minimization of criterion functions, which ensure minimization of unbalance of phase powers and currents at the network input, and that of technical electricity losses in network and supply transformer substation. The obtained findings are aimed at creating more efficient information control systems by improving conventional AMCS systems that belong to the category of information and measurement systems.

\section{REFERENCES}

[1] Yakushkov K.V. "Automated system of the fiscal electricity metering for the retail market," Informatizatsiya i sistemy upravleniya v promyshlennosti, 2009, no. 3 (23), pp. 9-13 (in Russian).

[2] El-Hawary, M.E. "The Smart Grid-State-of-the-art and Future Trends," Electr. Power Compon. Syst. 2014, 42, pp. 239-250.

[3] Kosoukhob F.D., Vasilev N.V., Filipov A.O. "Reducing losses from current unbalance and improving electricenergy quality in $0.38-\mathrm{kV}$ networks with municipal loads", Russian electrical engineering, Allerton Press, Inc. (New York), vol. 85, no. 6, 2014, pp. 350-353.

[4] Omorov T.T. "AMCS-based assessment of the current and voltage unbalance effect on power losses in the distribution grid," Elektrichestvo, 2017, no. 9, pp. 1723 (in Russian).

[5] Czarnecki L.S., Bhattarai P.D. "Currents' physical components (CPC) in three-phase systems with asymmetrical voltage," Przegląd Elektrotechniczny, 2015, no.6, pp. 40-47. DOI: 10.15199/48.2015.06.06.

[6] Ponomarenko O. I., Holodinov I.H. "Effect of unbalanced conditions on power losses in power grids of distributed power supply systems," Energetik, 2015, no.12, pp. 6-8 (in Russian).

[7] Mahmoud, K.; Yorino, N.; Ahmed, A. "Optimal Distributed Generation Allocation in Distribution Systems for Loss Minimization," IEEE Trans. Power Syst. 2016, 31, pp. 960-969.

[8] Harutyunyan A.G. "On the calculation of additional power losses in three-phase four-wire grids," Elektrichestvo, 2015, no.10, pp. 55-58 (in Russian).

[9] Averbukh M.A., Zhilin E.V. On the loss of electricity in power supply systems for individual housing construction. Energetik. 2016. no. 6. pp. 54-56 (in Russian).

[10] Samokish V.V. Patent No. 2548656 (Russian Federation). "Method of balancing phase currents of three-phase four-wire line and device for its implementation," Bull. no.11. 20.04.2015 (in Russian).

[11] G.A.Bolshanin. "Patent No. 2249286 (Russian Federation). Method for computer-aided online measurement of current and voltage unbalance level, Bull. no.9. 28.03.2005. (in Russian)

[12] I.V.Naumov, D.A.Ivanov, S.V.Podjachikh. Patent No. 2490768 (Russian Federation). Balancer for three-phase networks with neutral wire. Bull. no. 23. 21.08.2013 (in Russian).

[13] Kiselyov M. G., Lepanov M.G. "Balancing of currents in electrical grids by a power electronic controller of non-active power." Elektrotekhnika, 2018, no.11, pp. 63-70 (in Russian).

[14] Omorov T.T. "Balancing of a power distribution network by digital control," Mekhatronika, Avtomatizatsiya, Upravlenie, 2018, vol. 19, no.3, pp. 194-200 (In Russian).

[15] Omorov T.T. "Synthesis of a digital controller for balancing the power distribution network," Instruments and Systems: Monitoring, Control, and Diagnostics, 2019, no. 11, pp.51-56 (in Russian).

[16] https://mir-omsk.ru/products/oborudovanie-dlyatsifrovykh-setey/umnye-schetchiki-elektroenergii/ .

[17] Tanveer A. "Non-technical loss analysis and prevention using smart meters," Renewable and Sustainable Energy Reviews. 2017. Vol. 72. pp. 573-589.

[18] "Methods of the classical and modern theory of automatic control: in 5 volumes", Ed. by K.A. Pupkova, N. D. Egupova. M.: Publishing House of the Bauman Moscow State Technical University, 2004. (in Russian)

[19] Yiu, Joseph. The Definitive Guide to the Arm Cortex-M3 / Joseph Yiu; translated by A. V. Evstifeev. Moscow: Dodeka XXI, 2012. 552 p.

[20] Developing a New Generation of Smart Automation with ARM Cortex-M3 (https://www.radiolocman.com/ review/article.html?di=134036).

[21] Demirchyan K.S., Nejman L.R, Korovkin N.V et al. Theoretical foundations of electrical engineering, vol.1. St.Petersburg: Piter, 2009, 512 p. (in Russian).

[22] Aoki M. Introduction to optimization methods. Moscow: Nauka, 1977, 334 p. (in Russian).

[23] Emeljanov S.I., Utkin V. I., Taran V. A. The theory of systems of a variable structure. Moscow: Nauka, 1970, 592 p. (in Russian). 


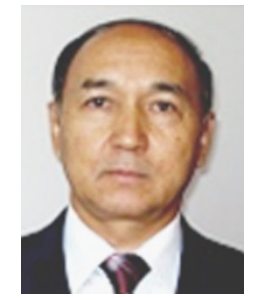

Turatbek Omorov is D.Sc. in engineering, Head of the Laboratory "Adaptive and Intelligent Systems" of the National Academy of Sciences of the Kyrgyz Republic (NAS KR), Bishkek. He is a Corresponding Member of the NAS KR. He graduated from the Faculty of Automation and Electrical Engineering of Leningrad Electrotechnical Institute in 1975. T. Omorov received his Ph.D. degree in engineering from the Bauman Moscow State Technical University in 1981 and a D.Sc. degree in engineering from the Scientific and Production Association "Cybernetics" of the Academy of Sciences of the Republic of Uzbekistan in 1997. He worked as the Director of the Institute of Automation (20002008), and Vice-President of the National Academy of Sciences of the Kyrgyz Republic (2008-2013).

Key research areas are automatic control, informatization and optimization of control processes, and automation of power systems.

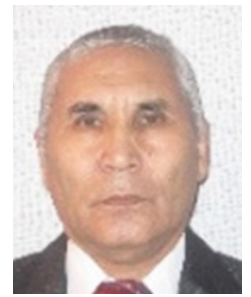

Takyrbashev Beishenaly is a Ph.D. in engineering, a Senior Researcher at the "Adaptive and Intelligent Systems" Laboratory at the NAS KR, Bishkek. He graduated from Frunze Polytechnic Institute in 1973 with a major in automation and remote control. He was the Head of the Power Systems Protection Service at JSC «Severelectro».

Key research areas are automatic control and automation of power systems.

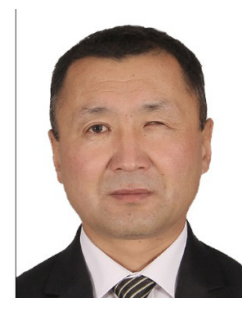

Kubanychbek Zakiriaev is a senior lecturer at Issyk-Kul State University, Karakol, Kyrgyz Republic. He graduated from the Faculty of Automation and Computer Engineering of Tomsk Polytechnic University in 1992.

Key research areas are information technology and automation of power systems

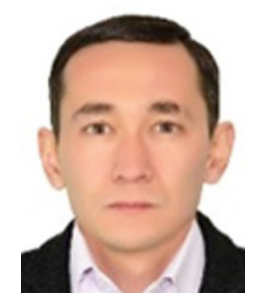

Taalaibek Koibagarov is a Ph.D. student at NAS KR, Bishkek. He graduated from the Faculty of Energy of the Kyrgyz Technical University in 2002. He is the Head of the Metrological Service of JSC "Severelectro."

Key research areas are automatic control and automation of power systems. 